\title{
DESHIDRATACION AGUDA Y HEMATOMA SUBDURAL EN EL LACTANTE
}

\author{
Drs. CLAUDE GODARD y JAVIER COX
}

Cátedra di Pediarria del Prof. Aniha! Ariztia, Hospital "Luis Callo Mackenna". Santiago.

El HSD del lactante es un proceso patológico cuya etiología queda a menudo obscura. Se ha mencionado como causas de él: traumatismo neonatal, carencias vitamínicas, distrofia, infecciones meníngeas, trastornos de coagulación sanguínea, pero estos factores no siempre es fácil determinarlos.

Ha sido sólo recientemente que algunos autores han sugerido la posibilidad de que una deshidratación aguda grave, pueda provocar un HSD. Herzberger (1956) estudió 33 casos de HSD en lactantes y encontró el antecedente de gastroenteritis aguda grave en 12 de ellos. Freudlich (1956) cita la diarrea con deshidratación como factor etiológico en 4 casos sobre 19 estudiados. Existen en la literatura médica varios otros informes de casos aislados 5-9-11-15-16-1a. En cambio otras estadísticas, más antiguas, no mencionan este factor etiológico ${ }^{10}$.

Más sugestiva nos parece la comprobación de derrames subdurates provocados en el curso de deshidrataciones hipernatrémicas en animales de experiencia jóvenes. Estos trabajos fundamentales de Girard T (1956) y Finberg ` (1959) nos indujeron a presentar los casos clínicos, en que creemos que esta relación existe.

Aprovechamos esta oportunidad para revisar la literatura existente sobre este tema $\mathrm{y}$ discutir el mecanismo de producción. la sintomatología, el diagnóstico y el tratamiento.

\section{PRESENTACIÓN DE CASOS}

CASO Nel 1: Fs un lacrante de 3 mescs. con anteidentes personales $y$ familiares sin imporancia, hospila'izado el 16-X-59 por presentar desde 3 dias, diarru y vómitos. luego fiebre e irritabiuidad.

Al ingreso, se comprueba lactapte febril, olnubi. lado, con gran deshidratación de piel y mucosas. breg. ma deprimidu, con colapso cardiovascular. Se diagnoslica toxicosis y se inicia tatamiento con fleboclisis (suiro 3: 2: 1), antibióticos y dięta hidrica. lin el Servicio el niño mejora al dia siguiente. para igravarse de nuevo al cercero, presentando convalsiones repetidas. Fo los dias siguientes pasa progtesiva. mente de un estado soporoso a coma profundo. pese a la ancibiocerapia, oxigeno, fleboclisis con solución Darrow, glucosada y plasnia. Persisten los signos de deshidratación celular, con mucosas muy secas, pie: empastada; diuresis buena; edemas maleolares. Neuralógicamente presenta hipotonía muscular, teflejos vivos, ristagmus horizontal, desviación de ambos ojos hacis la derecha, después hacia la izquierda. La punción lumbar da salida a líquido claro. a presión disminuida. con $1.5 \mathrm{gr}$ de albúmina y $1.47 \mathrm{gr}$ de glucosa. siendo el resto normal. Los ionogramas dan los resultados indicados en el zuadro $N^{0} 1$.

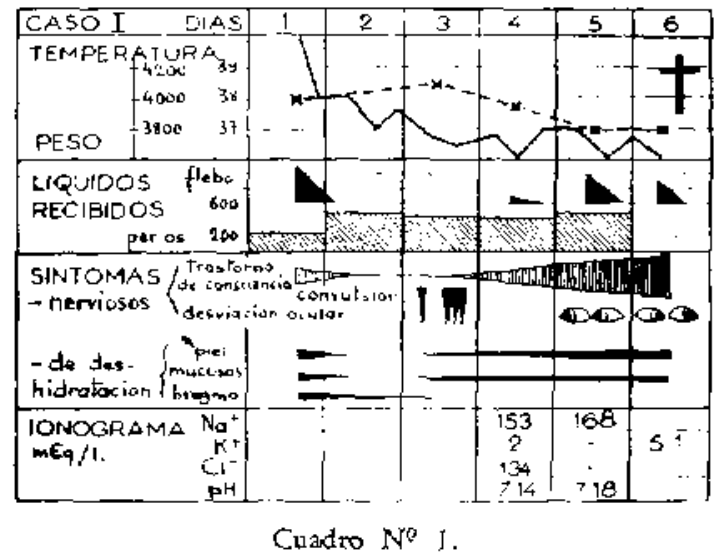

Fallece al $6^{\circ}$ día de hospitalización.

En la autopsia se comprueba hígado graso. lesiones atelectásicas de los pulmones, moderada hiperemia cerebral y sobre todo un hematoma subdura! laminar sobre el hemisferio izquierdo, considerado de carácres ruciente for cl anatomopatólogo (fig. 1).

En resumen: Se trata de un lactante menor que presenta, a raíz de un trastorno nutritivo agudo, una deshidratación persistente, con curva de peso estacionaria, agravación de la deshidratación celular e hiperhidratación extracelular secundaria (edemas).

Desde el punto de vista metabólico: hipernatremia, hipercloremia, acidosis, 


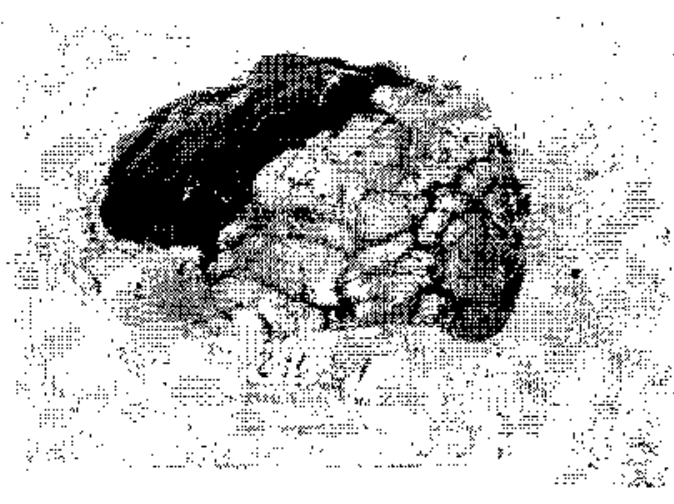

Fïigura Ne 1. - Hematoma subdutal sobte e1 hemisferio izquierdo, en el caso $\mathrm{V}^{\mathrm{o}} \mathrm{l}$.

Graves sintomas de daño cerebral: coma, convulsiones y trastornos respiratorios que lo llevaron a la muerte.

Hematoma subdural izquierdo: por su aspecto muy reciente y la evolución clínica especial después de un cuadro de toxicosis corriente, el que nos parece con más probabilidad secundario al trastorno metabólico descrito.

CASO N 2: Es un lactance de 6 meses. Ancece.dentes familiares y personales sin importancia. HospiLalizado el $23-I I-60$ por presentar desde una semana diarrea, luego vómitos e inapetencia.

Al ingreso se comprueba lactante en mal estado general $y$ nutritivo, abnubitado, con gran deshidratación de piel y macosas, bregma deprimido. ojos hundidos. respiratión profunda. La pérdida de peso sc calcula a màs de $12 \%$ en 7 dias. Con el diagnóstico de distrofia $y$ toxicosis se inicia tratamiento con fleboclisis (sucro Ringer-glucosado), cloramfenicol y dien ta bidrica.

Fn el Servicio el niño presenta al dia siguiente una ligera mejoria, para caer en estado agónico al tercero,

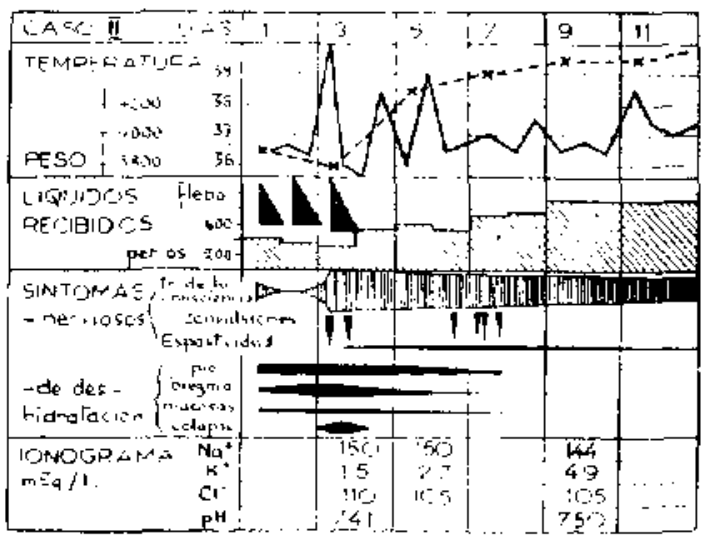

Cuadro $N^{-0} 2$. con colapso cardiorascular intenso, deshidratación marcada, facies tóxica, coma, bipertermia, convulsiones generalizadas repetidas. Llama la atención un bregma sumatnente deprimido y ausencia de lCR en la punción umbat. Se rehidrata cn forma intinsiva con plasma, suero glucosalino y Darrow. En los días siguientes c] riño corrige su trastorno metabólico. sube de pesa. pero persiste en coma y muestra rigidez de descirebración. Los ionogramas dan los tesultados indicados en los cuadros. Al electrosardiograma, signos eléctricos sugestivos de hipopotasemia.

E: quinco dia se practica punción subdura. que fue negativa, y punción ventricular que da salida a liquido zantocrómico a muy exasa presión, con $1 \mathrm{gr}$ de albúmina, el resto normal.

Una semana despuks, una thueva expioración sub. dural da salida a abundante líquido xantocrómico turbio en ambos lados. Con el diagnóstico de HSD. so inicia el tratamiento de punciones evacuadoras alternadas. Con la progresión de este método se va notando una gran mejoría de su cuadro neuro'ógico, recupeta paslutimmente la conciencia. desaparece la hipertonia muscular. Un mes despuśs al niño tiene un comporramiento normal, sonrie, etc. La exploración quirúr sica bilatetal a savés de agnjeros de trepanacion demastró la existencia de sendos HSD, con memb:ana bien constituída.

In electroencefalograma hecho nta semana despućs del ingreso muestra abolición de actiridad cortical electrica, con ondas lentas de bajo voltaje Repetido un mes $y$ medio después. el examen nues[r] udil normulización casi completa del trazado.

En resumen, se trata de un lactante distrófico que presenta, a raíz de un trastorno nutritivo agudo, una deshidratación grave y persistente y desde el punto de vista metabólico: hipernatremia e hipokalemia. Presentó secundariamenter marcados signos de daño cerebral difuso: coma, convulsiones que, juntos con la ausencia de líquido, en la punción lumbar, el bregma muy deprimido y la hipotensión ventricular hicieron pensar en un síndrome de colapso cerebral.

Los sintomas neurológicos persistieron después de la corrección de la deshidratación y de la recuperación del peso.

Al $10^{\circ}$ día se comprobó HSD bilateral, en circunstancias de que una semana antes la punción exploradora era negativa.

\section{DISCUSIóN}

Basados en el análisis de nuestras dos observaciones y de los casos de la literatura, vamos a discutir la etiología, la pa- 
tologia, la sintomatologia, el diagnóstico y el tratamiento de estos HSD.

Etiología. Es bien sabido que un cuadro de deshidratación aguda grave en un lactante (toxicosis) es capaz de provocar un daño cerebral difuso. El daño es funcional, y en la inmensa mayoría de los casos pasa rápidamente con el tratamiento adecuado del trastorno metabólico. Excepcionalmente este daño cerebral podría convertirse en orgánico, dejando secuelas a veces definitivas.

En segundo lugar, las experiencias de Girard ${ }^{3}$ y Finberg " parecen demostrar que una deshidratación hipernatrémica puede provocar hemorragias subdurales. Estos autores provocaron sobrecarga de cloruro de sodio en gatos jóvenes sometiéndolos además a privación de agua. Girard comprobó en los 15 gatitos de su experiencia, hemorragias subdurales, $y$ en seis de los 15, discretas hemorragias en otros órganos (peritoneo, hígado). Finberg ${ }^{5}$, por su parte, encontró hemorragias subdurades en 23 de 27 animales; en algunos de ellos existían además, pequenas lesiones hemorrágicas intracerebrales. Menciona además el caso clínico de un lactante de 5 semanas que por error, fué sometido a un aporte excesivo de sal que se calculó en 30-40 gr en 12 horas. Este paciente desarrolló el cuadro de una deshidratación hipernatrémica típica, y posteriormente quedó con secuelas neurológicas graves imputables a un HSD.

Por otra parte, es importante recordar que un enfermo con una lesión previa del sistema nervioso central (encefalitis, HSD) puede desarrollar en ciertos momentos de su evolución una deshidratación secundaria. En efecto, un trastorno profundo de conciencia impide las manifestaciones de sed y puede conducir a una privación de agua, provocando una deshidratación con hipernatremia (Welt). Esta hipernatremia quizás padría también ser causada por un daño electivo de los centros osmoreceptores del sistema hipotálamohipofisiario (Engel ${ }^{3}$, Peter ${ }^{14}$ ).

El problema más importante que se presenta ante el análisis de uno de estos cuadros es, por to tanto, el poder dilucidar en forma clara qué es lo primero: la deshidratación o el HSD. Inclusive en situaciones en que la deshidratación aguda aparece como el primer signo de enfermedad, se puede plantear la duda de que el hematoma pudiera haber existido ya antes, aunque asintomático.

En nuestros dos casos, nos parece que el HSD siguió al proceso de deshidratación. En ninguno de los niños había signos previos que hicieran pensar en un daño cerebral (parto y desarrollo normales, etc.). En la autopsia del primer caso el anatomopatólogo informó la hemorragia subdural como de carácter muy reciente. En el $2^{9}$ caso la exploración subdural bilateral al $4^{\circ}$ día de evolución fué negativa, en cambio la repetición de este procedimiento una semana después demostró en forma indudable la existencia de HSD bilateral importante.

Patogenia. Varios son los factores a los cuales se ha atribuído importancia en la producción de hemorragia subdural durante una deshidratación aguda.

Se ineriminó a la punción subdural misma. Herzberger ${ }^{8}$ piensa que el peligro consiste en la repetición de las punciones después del fracaso de una primera, pero recalca que en la mayoría de los casos ya la primera punción es positiva. Freundlich ${ }^{6}$ cree que la punción subdural podria provocar una hemorragia en lactantes con tendencia hemorragípara clínica o subclínica. Es interesante recordar que $\mathrm{Ma}$ toth ${ }^{13}$ demostró la existencia de hipoprotrombinemia en la toxicosis.

Girard ${ }^{7}$ sugiere que la producción de HSD estaría en relación con la disminución del volumen del cerebro, o colapso cerebral. Este fenómeno se ha comprobado repetidamente en operaciones quirúrgicas para HSD de origen traumático en el adulto, y según algunos la contracción del cerebro no sería debida a la compresión por el hematoma, sino sería primaria y la hemorragia en la duramadre su consecuencia (Lazorthes ${ }^{12}$, Chavany ${ }^{8}$ ). En el lactante, un grado leve o mediano de hipotensión intracraneana es de comprobación frecuente durante la toxicosis ( fontanela deprimida, líquido cefaloraquídeo a escasa presión) muy acentuada como en el caso 2 ella podría explícar el "estado cerebromeníngeo" de algunas toxicosis acompañadas de coma, convulsiones e hipertermia, Sorrel ${ }^{17}$. Desde un punto de vista teórico se puede hacer una distinción entre un colapso cerebral debido a contracción ventricular y disminución de la presión del LCR, que corresponde a una deshidratación extracelular 
( el LCR haciendo parte de este sector colapso ventricular), y otro producido por disminución del volumen de la masa cerebral misma, correspondiente a una deshidratación celular (colapso cerebral verdadero). Prácticamente, estas dos formas pueden reunirse.

Girard ${ }^{7}$ provocó experimentalmente estos dos tipos de colapso cerebral, el primero por substracción de LCR, y el segundo por inyección de cloruro de sodio hipertónico. En el primer caso no produjo lesiones, pero sí en el caso de colapso cerebral verdadero. Sin embargo, varios autores creen que un colapso ventricular sólo es capaz de provocar hemorragias subdurales. Girard ${ }^{7}$ sugiere el mecanismo siguiente para explicar la formación del hematoma: la disminución del volumen del cerebro produciria un tironeamiento de las venas-puente que desde el cerebro móvil se dirigen, atravesando el espacio subdural, hacia la duramadre adherida a la caja craneana fija. Esto provocaría ruptura de algunas de estas venas en dicho espacio y la formación progresiva del hematoma.

Finberg fué el primero en mencionar la importancia de la hipernatremia, en las manifestaciones neurológicas de la deshidratación aguda del lactante, y en las alteraciones del liquido cefaloraquídeo, y en demostrar que la deshidratación hipernatrémica puede provocar un daño cerebral anatómico, especialmente bajo la forma de hemorragias en la duramadre. Es importante recalcar que en todos los casos de la literatura donde el ionograma fué determinado (Finberg, Pringuet, Tuxpijn), la hipernatremia fué un hecho constante. El modo de actuar de la hipernatremia no está aún bien establecido. El ión sodio podría tener una acción hemorragípara propia, pero si éste fuera el mecanismo sería lógico encontrar hemorragias en diversos órganos, cosa que no ha ocurrido. Más convincente parece la hipótesis siguiente: la hipernatremia provoca un movimiento de agua fuera de la céIula para restablecer el equilibrio osmótico entre los espacios intra y extracelulares. Se crea así un estado de deshidratación celular. Esta situación es la que en el cerebro produciria la disminución de volumen de la masa cerebral. Un hecho sugiere que este fenómeno de deshidra- tación celular sería más marcado para la célula nerviosa que para otras células del organismo: Finberg i mostró experimentalmente que, a diferencia de lo que sucede en el resto de las células, el sodio es incapaz de penetrar a la célula nerviosa, para restablecer el equilibrio osmótico perdido por el exceso de concentración iónica en el espacio extracelular. Al parecer, no siempre la hipernatremia provoca hemorragias, sino que en algunos casos, lo que nos induce a pensar que aún quedan muchos puntos obsciuros en la patogenia de este proceso.

Sintomatologia. Estudiado los casos de la literatura y los nuestros, hemos sacado cono primer hecho claro que en ninguno de ellos hubo signos y síntomas neurológicos que pudiéramos llamar sugestivos de HSD (cuadro $\mathrm{N}^{0} 3$ ).

CUADRO No 3

SINTONAS NEUROLOGICOS

En 13 casos de deshtdrataclon aguda grave con hematoma stibdiral secundario:

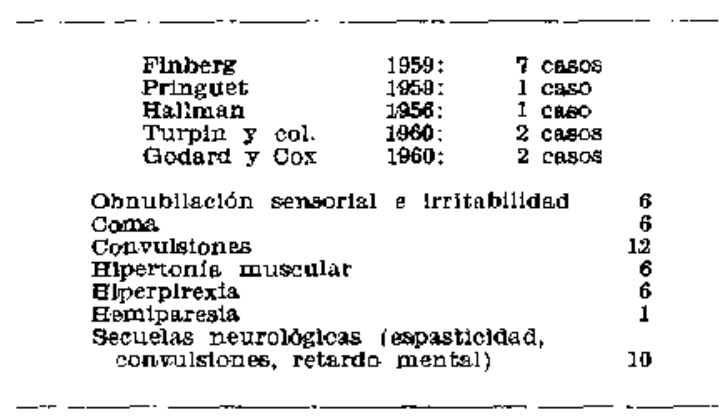

Por el contrario, la sintomatología casi siempre correspondió a manifestaciones de un daño cerebral difuso, trastorno de la conciencia, irritabilidad, hipertonía muscular, hiperpirexia. Vale la pena recordar que estos signos son justamente los que se consideran tipicos de la carencia primaria de agua, o sea de una deshidratación hipematrémica verdadera.

Sólo un caso de Finberg ${ }^{5}$ tuvo hemiplegia. En dos enfermos hubo convulsiones localizadas a un lado, pero en lactante este tipo de convulsiones no tiene ni con mucho el valor de localización que se les atribuye en el adulto.

En todo caso, a nuestro parecer, el HSD producido en estas circunstancias se manifiesta clínicamente en forma más violenta y difusa que el HSD crónica ("trau- 
mático") del lactante, en el cual la sintomatología nerviosa es más lenta y menos intensa en su aparición.

En segundo lugar, las manifestaciones del daño cerebrai difuso han continuado en todos los casos pasado el periodo agudo de la enfermedad, y algunos quedaron con verdaderas secuelas neurológicas. En resumen, el hecho sugestivo de la presencia de un derrame subdural, fué no el carácter de la sintomatología neurológica, si no la duración anormal de ella.

\section{HALLAZGOS DE LABORATORIO}

Ionograma. Hecho constante en los casos estudiados en este sentido fué una hiperelectrolitemia con concentración alta de ión sodio en la sangre y LCR. No siempre se podrá encontrar cifras muy altas en todas las circunstancias, ya que no existe un paralelismo exacto entre la evolución del trastorno metabólico y del fenómeno hemorrágico que éste provocó. Mientras el primero podría haberse modificado con adecuada terapéutica, la lesión orgánica puede seguir evolucionando en forma arrastrada para manifestarse clínicamente cuando la natremia ya está normal.

Líquido cefaloraquideo. Puede encontrarse aumento de la cifra de albúmina (como en nuestros casos). En opinión de Finberg ", este hecho encontrado frecuentemente en la deshidratación hipernatrémica, es un índice sugestivo de hemorragia en el SNC.

Electroencefalograma. No parece aportar datos específicos en la etapa aguda de la enfermedad. En cambio, en la etapa de convalescencia o de secuelas neurológicas, puede dar signos más o menos indicativos de acúmulo de líquido en el espacio subdural: disminución del voltaje, con o sin actividad lenta difusa, hasta el grado de llegar a verdadero silencio eléctrico, como sucedió en nuestro segundo caso.

La punción subdural. Tiene un valor diagnóstico fundamental. Su técrica es sencilla e inofensiva para el paciente cuando se hace bien. El aspecto del líquido extraido es semejante al de cualquier otro tipo de HISD. Puede tener un aspecto de sangre reciente, dado el hecho de que su evolución es más corta (punción más precoz).
Debemos recalcar que siempre la exploración subdural debe ser bilateral, haya a no signos de localización. Recomendamos también que esta exploración se haga pasado el período agudo de deshidratación de la enfermedad.

Finalmente la conducta que debe seguirse en estos casos será la misma que en todo paciente en que se diagnostique HSD, y el tratamiento será igual.

\section{RESUMEN Y CONCLUSTONES}

Se presentan dos casos de HSD en lactantes en los cuales se presume que la causa de ellos fué una deshidratación aguda hipernatrémica. Uno de ellos fué comprobado en Ia autopsia, y el otro por exploración subdural.

Creemos que estos casos, y los que hemos revisado en la literatura, permiten sacar las siguientes conclusiones:

Un trastorno nutritivo agudo es capaz. de producir un daño cerebral orgánico grosero del tipo de la hemorragia subdural. Este trastorno sería del tipo de la deshidratación hipernatrémica.

En toda toxicosis o cuadro que la semeje, en que el trastorno de conciencia y los signos nerviosos no tienen la evolución favorable corriente, y al contrario evolucionan en forma persistente hasta llegar incluso a provocar secuelas, se justifica la investigación de posible HSD.

Creemos necesario recalicar la necesicad de analizar cada caso en forma escrupulosa para poder dilucidar cual es el hecho primitivo: HSD o trastorno meta. bólico agudo.

Por las características clínicas de este tipo especial de HSD, con evolución violenta y rápida y manifestaciones de daño profundo y difuso de SNC, nos parece justificado, y así lo proponemos, denominar a esite cuadro "Hematoma subdural agudo del lactante, secuela de deshidratación hipernatrémica".

\section{SUMMARY}

\section{SUBDURAL HEMATOME IN TOXICOSIS}

Two cases of Subdural Hematome in infants in which it is presumed that the cause was acute hypernatremic dehidrat. ion, are presented. 
One of them with necropsy verification, and the other by subdural puncture.

We believe that these cases, and those we have revised in literature, let us come to the following conclusion:

Acute toxic diarreha is capable of producing heavy organic cerebral injury of subdural hemorrage type. This disturbance would of hypernatremic dehydration type.

In all toxic or similar condition in which conscience is lost, and all nervous signs do not have the ordinary favorable course and on the contrary they persist reaching the form of sequels, the investigation of a possible Subdural Hematome is justified.

We would like to call the attetion upon the necessity of analizing every case, to be able to determin which is the primary cause: subdural hematome or acute methabolic disfunction.

By the clinical characteristics of this special type of hemorrage, with rapid and violent evolution and deep and difuse injury of the Nervous Central System, we belive it reasonable, and therefore we propose it, to call this condition acute subdural hematome of the infant, caused by hypernatremic dehydration.

\section{ZUSAMMENFASSUNG}

\section{AKUTE WASSERVERARMUNG UND SUBDURALES HAEMATOM BEIN SAUGLING}

Zwei Fälle von subduralem Haematom bei Säuglingen werden vorgestellt, bei denen vermutlich akute hypernatriaemis che Wasserverarmung die Ursache war. Einer der Fälle wurde bei der Sektion bestätigt und der andere durch subdurale Untersuchung.

Wir glauben, dass diese Fälle und diejenigen, die wir bei Durchsicht der Literatur gefunden haben, die folgenden Schlussfolgerungen zu ziehen gestatten:

Eine akute Ernährungsstörung ist imstande, eine grobe organische Hirnschädigung nach Art der subduralen Blutung hervorzurufen. Diese Störung dürfte ihrer Art nach eine hypernatriaemische Wasseaverarmung sein.

Bei jeder Toxikose und bei jedem ihr ähnlichen Krankheitsbild, bei dem die nervösen Erscheinungen nicht den üblich- en günstigen Verlauf nehmen, vielmehr sich unaufhaltsam weiterentwickeln, bis sie schliesslich Folgezustände herbeiführ. en, ist die Untersuchung auf ein mö gliches subdurales. Haematom gerechtfertigt.

Wir halten es für nötig, die Notwendig. keit zu betonen, dass jeder Fall sorgfältig analysiert wird, um aufzuklären, welches das ursprüngliche Ereignis ist: das subdurale Haematom oder die akute Stoffwechseltörung.

Wegen der klinischen Besonderheiten dieses besonderen Typs von subduralem Haematom mit seinem heftigen und raschen Verlauf und den Erscheinungen einer tiefgreifenden und diffusen Schädigung des Zentralnervensystems scheint es uns gerechtfertigt und schlagen wir vor, dieses Krankheitsbild "akutes subdurales Haematom des Säuglings infolge hypernatriaemischer Wasserverarmung" zu benennen.

\section{BIBLIOGRAFIA}

1. ANDERSON, F., $M_{1}-$ subdural hematoma, a complication of operation for bydrocephalus. Pediatrics. 10:11, 1952.

2.-CHAVANY, J. A. - Coma ainguller, collepsus cerebral spontane, hematome solis-dural secondelre. Presse Med. 61:112, 1953.

3.-ENGEL, F. I.; JAECGER, C. - Dehydratation with byperzatremia complicating nasogratslc tube feeding. Am. J. Med. 17:196, 1054 .

4.-FIABERG, L.; HARRISSON, H. F. - Hypernatremia in infants. Pediatrics. 16:1, Ins5.

5.-FINBERG, L. - Pathogenesis of lestons of the neroous system in hypernatremle states. Pediatrics. $23: 40,1959$.

6.-FREUNDLICH, E. - Shubdural hematoma in infancy. Am. J. Dis. Conlda. al-608, 1056.

7.-GIRARD, F. - Les hématomes sous-duraux: étude expérimentale. Acta, Paediatr. 45:618, 1954.

8.-HERZBERGFR, F. $\longrightarrow$ Remarkes of thirty three easee of subdural effuslons in infancy. Arch. Dis. Chlla. 31:44, 1056 .

9.-HALLMANN, N. - Ueber zerebrale Schaedigutigen newch gastroenteritis im Soeuglingsalter. Ann. Paed. Feñ. $2: 3 I_{1}, 1956$.

10.-INGRAHAM, F. D.; MATSON, D. D. $\rightarrow$ Subdurai hematoma in infancy. Advances in Paed, 4:231. 1949.

11.-LELONG, M. - Hemetome sous-dural chropique du nourrisson. Anch. Fr. Ped. 12:1037, 1955.

12.--IAZORTHFS, G. - Lhypotension cephalorachidienna et l'hematome sous-dural. Ret. Neurol. B5: 413, 10951.

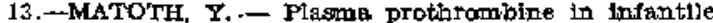
diarrhea. Am. J, Dis. Child. 80:944, 1950.

14. -EETHRS, J. P.; WHT, L. G. - A salt wasting syndrome assoclated with cerebral disease. Tr. A. Am. Phys. 63:57, 1850.

15. -DE PAIVA, S. L.; BARROS, F. P. - Derrames subdursis na infencha. Pediatr. Prat. Bradl. 26:59, 1955.

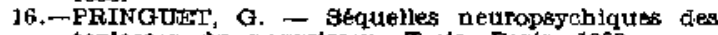
toxlcoses du bourrissom. Teols, Paris. 1959.

1't. - BORRFI, R. - Ite collapsus ostebraux ches I'enfant, Arch. Fr. Ped. 9:781, 105e.

18. - TUUTERI, L. $\rightarrow$ On the circulation time and the oxygen satiaration sinus blood in severe infantlle gastroenterltis. $42: 14,1953$.

19.-TDRPIN, R.i GORIN, $\mathbf{R}$. - Les Hematome sousduraux au cours des deshydratations hypernatres. miques du nourrisgon. \$. H. P. (ennsles de pédlatrie) (en preparación) 1060. 